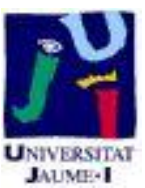

Título artículo / Títol article: Theoretical study of primary reaction of Pseudozyma antarctica lipase B as the starting point to understand its promiscuity

Autores / Autors

Katarzyna Swiderek, Sergio Martí, Vicent Moliner

Revista:

ACS Catalysis, 2014, 4 (2)

Versión / Versió:

Postprint de l'autor

Cita bibliográfica / Cita

SWIDEREK, K.; MARTÍ FORÉS, S.; MOLINER bibliogràfica (ISO 690): IBÁÑEZ, V. Theoretical study of primary reaction of Pseudozyma antarctica lipase B as the starting point to understand its promiscuity. ACS Catalysis, 2014, 4 (2), pp 426-434 


\title{
Theoretical study of primary reaction of Pseudozyma antarctica lipase B as the starting point to understand its promiscuity
}

\author{
Katarzyna Świderek, ${ }^{[b][c]}$ Sergio Martí ${ }^{[a]}$ and Vicent Moliner* ${ }^{[a]}$
}

\begin{abstract}
Pseudozyma antarctica lipase B (PALB) is a serine hydrolase that catalyzes the hydrolysis of carboxylic acid esters in aqueous medium but it has also shown catalytic activity for a plethora of reactions. This promiscuous activity has found widespread applications. In the present paper, the primary reaction of PALB, its native hydrolytic activity, has been studied using hybrid quantum mechanical/molecular mechanical (QM/MM) potentials. Free energy surfaces, obtained from QM/MM Molecular Dynamics (MD) simulations,
\end{abstract}

show that the reaction takes place by means of a multi-step mechanism where the first step, the activation of the carbonyl group of the substrate and the nucleophilic attack of Ser105 to the carbonyl carbon atom, presents the highest energy transition state. Our results, which are in good agreement with kinetic experimental data, suggest that the origin of the catalytic activity of the enzyme is due to favorable interactions established between the residues of the active site that create an oxyanion hole, Gln106 and Thr40, as well as the Asp187 that is capable of modulating the pKa of His 224 to act as a base or an acid depending on the step of the catalytic process. Our results can be used to rationalize the design of an optimum biocatalyst to accelerate fundamental reactions in organic synthesis based on the protein scaffold of PALB.

Keywords: Hydrolysis $\bullet$ Enzyme
promiscuity $\bullet$ Pseudozyma
antarctica lipase B $\bullet$ Candida
antarctica lipase B $\bullet$ Kinetic isotope
effects $\bullet$ QM/MM

\section{Introduction}

During the past decades, the possibility of modifying natural existing enzymes has attracted the attention of academia and industry since it can open the door to obtaining new biocatalyst that present high efficiency, selectivity, fewer unwanted side-products,

[a] Dr. Sergio Martí, Prof. Vicent Moline

Departament de Química Física i Analítica

Universitat Jaume I

12071 Castellón, Spain

Fax: (+34) 964-728066

E-mail: moliner@uji.es

[b] Dr. Katarzyna Świderek

Departament de Química Física

Universitat de València

46100 Burjassot, Spain

[c] Dr. Katarzyna Świderek

Institute of Applied Radiation Chemistry

Lodz University of Technology

90-924 Lodz, Poland

Supporting information for this article is available on the WWW under http://www.chemeurj.org/ or from the author. the use of a one-pot procedure or the possibility of working at aqueous mild conditions of temperature or pressure. In this regard, nowadays the use of computer-based methods, in combination with in vitro directed evolution and labour-intensive screening of microbial cultures for the desired activities appears to be a promising tool. ${ }^{1}$ Different goals, apart from increasing or generating activity, have been achieved during the past years in the designing of new biocatalysts, such as altered substrate specificity, improved thermostability, organostability, regioselectivity, enantioselectivity, etc... ${ }^{2}$

Among all possible methods, apart from generation of the full protein machinery from scratch, or de novo protein design, that have shown outstanding but limited number of successful challenges, ${ }^{3,4,5}$ the re-design of a protein active site is one of the most promising strategies at present. In this technique, the cavity of an existing natural protein is modified by mutating a subset of its side chain amino-acids. The starting protein scaffold for the rational re-design of an active site can be a promiscuous enzyme $e^{6,7,8}$ an enzyme that presents the ability to catalyze, at lower efficiency, a reaction other than its cognate reaction that is maintained via selective pressure. This provides a raw starting point for the evolution of enzymes, as a new duplicated gene presenting low activity would be the germen for adaptive evolution. ${ }^{6}$

Pseudozyma (formerly Candida) antarctica lipase B (PALB) is a serine hydrolase belonging to the group of $\alpha / \beta$-hydrolases that catalyzes the hydrolysis of carboxylic acid esters in aqueous medium but it also shows catalytic activity as carboxylic acid esterases, thioesterase, peptidase, dehalogenase, epoxide hydrolase, 
halo peroxidase, and cleaving C-C bonds. ${ }^{9} 10$ This promiscuous activity makes PALB to be a good model to develop new biocatalysts. As a serine hydrolase, it has been proposed that catalysis proceeds by activation of the carbonyl of the substrate ester through an oxyanion hole made by two residues (Thr40 and Gln106), which would stabilize the oxyanion intermediate and the nucleophilic attack of a serine residue (see detail of active site in Scheme 1). ${ }^{11}$ This serine would be coupled to another two conserved residues thus forming a catalytic triad (Ser105, Asp187 and His224). ${ }^{12}$ Nevertheless, despite the amount of studies devoted to the use modified hydrolases to develop new applications, a detailed description of the molecular mechanisms of the primary activity of PALB remains unclear, which hamper a quicker development of more efficient biocatalysts based on this promiscuous enzyme.

The aim of the present study is to get a complete picture of the molecular mechanism of PALB when working as a serine hydrolase by means of molecular dynamics (MD) simulations based on hybrid quantum mechanics/molecular mechanics $(\mathrm{QM} / \mathrm{MM})^{13}$ potentials. Information derived from the study of the primary reaction will be used to know what residues can be kept and which ones should be mutated to improve its activity on other reactions.

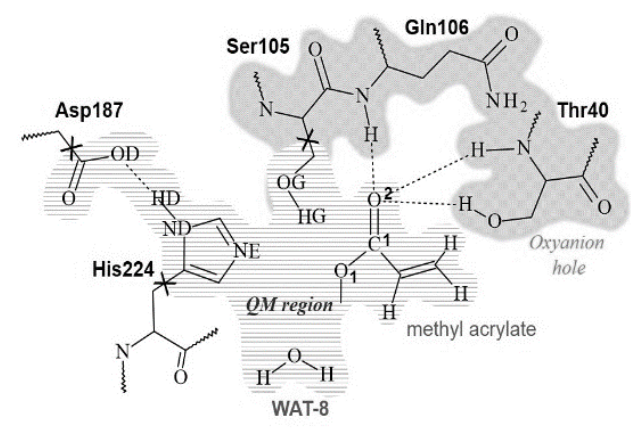

Scheme 1. Representation of the active site of PALB together with bound methyl acrylate substrate. Light grey shaded region contains atoms treated quantum mechanically. Oxyanion hole are highlighted in dark grey. Quantum link atoms are represented as crosses.

\section{Results and Discussion}

The exploration of the potential energy surfaces (PES) of every single possible step of the hydrolysis of methyl acrylate catalyzed by PALB (see Figure S1 of Supporting Information) has been used to compute the free energy surfaces in terms of 2D PMFs at M062X//OPLS/TIP3P level. The corresponding AM1/MM 2D PMFs are qualitatively equivalent to those presented in Figure 1 (see Figure S2 of Supporting Information). According to the surfaces presented in Figure 1, the reaction would take place through a multi-step mechanism equivalent to the ping-pong bi-bi reaction mechanism initially proposed by Hult and Berglund. ${ }^{12,14}$ Characterization of the stationary points located on the surfaces on Figure 1 has allowed proposing a step-wise mechanism as presented in Figure 2. Our results indicate that the hydrolysis of methyl acrylate catalyzed by PALB starts with the transfer of the hydroxyl proton of Ser105 to His224 and the concomitant attack to the carbonyl carbon atom of the substrate (see Figure 2). Once the substrate is covalently bounded to the backbone of the protein, intermediate 1, His224 acts as an acid by protonating the ester oxygen of the substrate $(\mathrm{O} 1)$ thus provoking the O1-C1 breaking bond and the methanol molecule release, intermediate 2. A conserved water molecule in the active site of PALB is responsible for the regeneration of the protonated His 224 and the nucleophilic attack to the carbonyl carbon atom of the substrate, intermediate 3 . Finally, a proton is transferred from the protonated His224 to oxygen atom of Ser105 that force the breaking of the substrate-protein O-C covalent bond. In this last step, the final product, acrylic acid, is obtained and the protein is
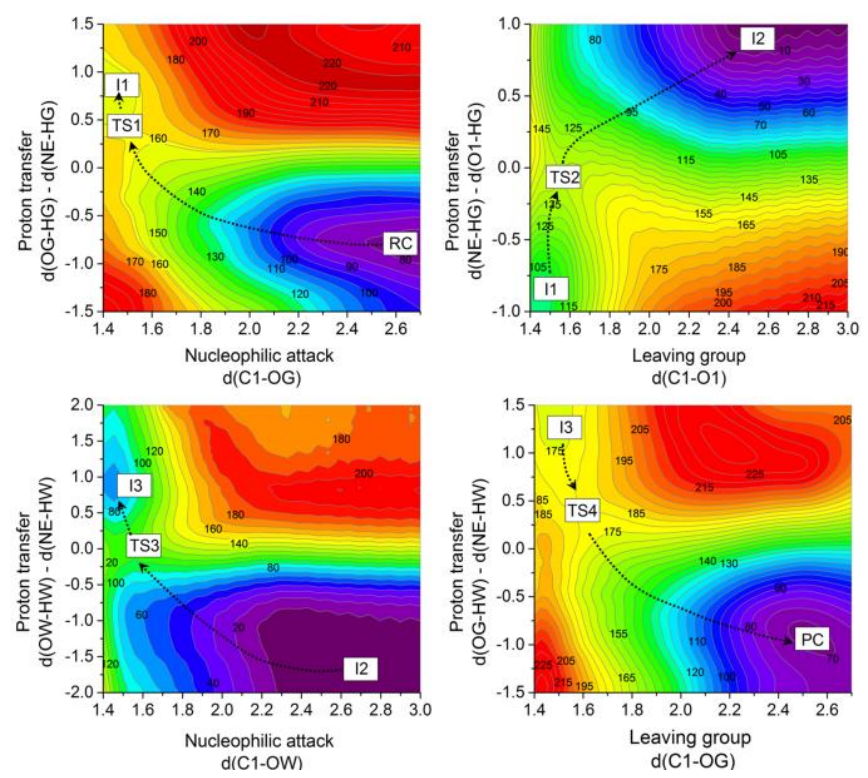

Figure 1. M06-2X//OPLS/TIP3P 2D-PMF surfaces for the reaction mechanism of hydrolysis of methyl acrylate catalyzed by PALB.

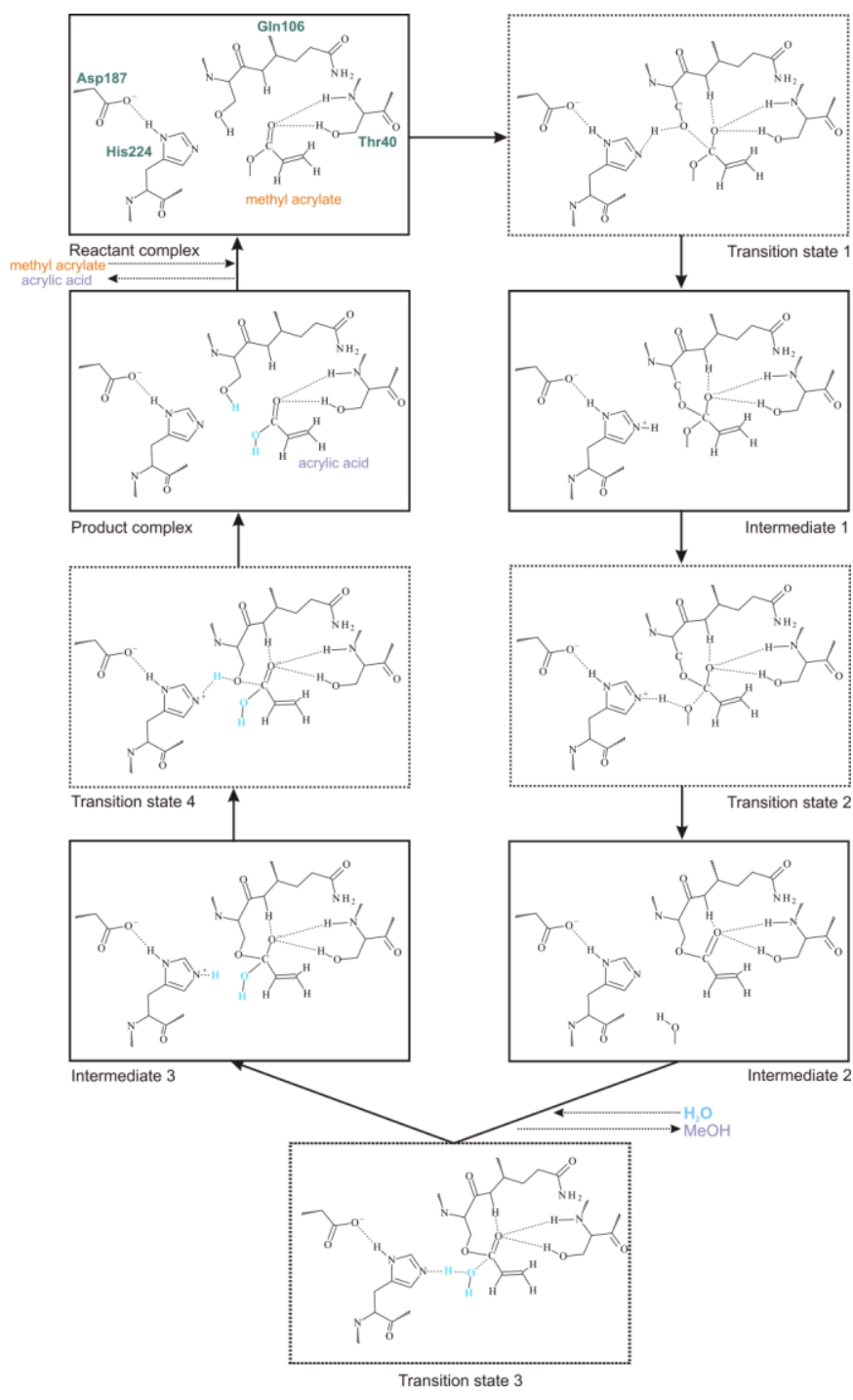

Figure 2. Multi-step proposed reaction mechanism for hydrolysis of methyl acrylate to acrylic acid and methanol, catalyzed by PALB. 
regenerated. As observed in the proposed mechanism some residues of the active site of the protein, apart from the mentioned His224 and Ser106, emerge as crucial for the catalysis.Thus, Asp187 appears to form a dyad with His224 modulating the pKa of this aminoacid that behaves as a base or an acid depending on the step of the reaction. This is confirmed by a geometrical analysis of the distance between both residues during the full process (see AM1/MM distances listed in Table 1).

Table 1. QM/MM averaged key distances (in $\AA$ ) obtained in the states located along the reaction path of the hydrolysis of methyl acrylate catalyzed by PALB.

\begin{tabular}{|c|c|c|c|c|c|c|c|c|c|}
\hline & RC & TS1 & I1 & TS2 & I2 & TS3 & I3 & TS4 & PC \\
\hline OG-HG & $0.97 \pm 0.02$ & $1.32 \pm 0.03$ & $2.15 \pm 0.03$ & $2.24 \pm 0.11$ & $3.05 \pm 0.21$ & $4.90 \pm 0.40$ & $4.68 \pm 0.21$ & $6.00 \pm 1.02$ & $2.97 \pm 0.33$ \\
\hline HG-NE & $2.25 \pm 0.03$ & $1.25 \pm 0.03$ & $1.02 \pm 0.03$ & $1.24 \pm 0.03$ & $2.58 \pm 0.03$ & $3.61 \pm 0.35$ & $3.44 \pm 0.21$ & $5.66 \pm 1.13$ & $2.74 \pm 0.27$ \\
\hline HG-O1 & $2.58 \pm 0.26$ & $2.26 \pm 0.11$ & $1.95 \pm 0.12$ & $1.32 \pm 0.03$ & $0.97 \pm 0.03$ & $0.97 \pm 0.03$ & $0.97 \pm 0.03$ & $0.97 \pm 0.02$ & $0.97 \pm 0.02$ \\
\hline $\mathrm{O} 1-\mathrm{C} 1$ & $1.37 \pm 0.02$ & $1.42 \pm 0.03$ & $1.46 \pm 0.03$ & $1.54 \pm 0.02$ & $3.08 \pm 0.02$ & $4.23 \pm 0.28$ & $4.09 \pm 0.15$ & $5.36 \pm 0.55$ & $3.39 \pm 0.23$ \\
\hline OG-C1 & $2.53 \pm 0.02$ & $1.53 \pm 0.02$ & $1.45 \pm 0.02$ & $1.42 \pm 0.03$ & $1.36 \pm 0.02$ & $1.43 \pm 0.02$ & $1.45 \pm 0.02$ & $1.55 \pm 0.02$ & $2.49 \pm 0.02$ \\
\hline $\mathrm{O}^{(\mathrm{HOH})}-\mathrm{C} 1$ & $4.14 \pm 0.32$ & $4.13 \pm 0.21$ & $4.06 \pm 0.26$ & $4.01 \pm 0.32$ & $4.65 \pm 0.77$ & $1.49 \pm 0.02$ & $1.44 \pm 0.03$ & $1.41 \pm 0.02$ & $1.36 \pm 0.02$ \\
\hline $\mathrm{H}^{(\mathrm{HOH})}-\mathrm{NE}$ & $5.16 \pm 0.83$ & $4.98 \pm 0.73$ & $4.20 \pm 0.36$ & $5.03 \pm 0.75$ & $5.17 \pm 0.71$ & $1.22 \pm 0.03$ & $1.03 \pm 0.03$ & $1.24 \pm 0.03$ & $2.46 \pm 0.03$ \\
\hline $\mathrm{H}^{(\mathrm{HOH})}-\mathrm{OG}$ & $5.84 \pm 0.75$ & $4.73 \pm 0.54$ & $4.30 \pm 0.31$ & $5.14 \pm 0.70$ & $5.65 \pm 0.77$ & $2.34 \pm 0.12$ & $2.39 \pm 0.18$ & $1.31 \pm 0.03$ & $0.97 \pm 0.03$ \\
\hline $\mathrm{C} 1-\mathrm{O} 2$ & $1.24 \pm 0.02$ & $1.29 \pm 0.02$ & $1.30 \pm 0.02$ & $1.29 \pm 0.02$ & $1.24 \pm 0.02$ & $1.30 \pm 0.02$ & $1.31 \pm 0.02$ & $1.29 \pm 0.02$ & $1.24 \pm 0.02$ \\
\hline \multicolumn{10}{|l|}{ His224-Asp187 } \\
\hline OD-HD & $2.37 \pm 0.33$ & $2.28 \pm 0.27$ & $2.22 \pm 0.18$ & $2.23 \pm 0.20$ & $2.37 \pm 0.23$ & $2.47 \pm 0.25$ & $2.31 \pm 0.20$ & $2.21 \pm 0.18$ & $2.35 \pm 0.22$ \\
\hline ND-HD & $0.99 \pm 0.02$ & $1.00 \pm 0.03$ & $1.01 \pm 0.03$ & $1.00 \pm 0.03$ & $1.00 \pm 0.03$ & $1.00 \pm 0.03$ & $1.00 \pm 0.03$ & $1.00 \pm 0.03$ & $0.99 \pm 0.02$ \\
\hline OD-ND & $3.11 \pm 0.22$ & $2.93 \pm 0.16$ & $2.98 \pm 0.13$ & $2.99 \pm 0.14$ & $3.13 \pm 0.16$ & $3.14 \pm 0.16$ & $3.05 \pm 0.14$ & $2.90 \pm 0.12$ & $3.10 \pm 0.17$ \\
\hline \multicolumn{10}{|l|}{ Oxyanion hole } \\
\hline $\mathrm{H}^{(\mathrm{G} \ln 106)}-\mathrm{O} 2$ & $3.70 \pm 0.15$ & $2.99 \pm 0.12$ & $2.95 \pm 0.11$ & $3.00 \pm 0.11$ & $3.01 \pm 0.14$ & $2.90 \pm 0.10$ & $2.87 \pm 0.10$ & $2.92 \pm 0.13$ & $3.46 \pm 0.20$ \\
\hline $\mathrm{H}^{(\mathrm{N}-\mathrm{Thr} 40)}-\mathrm{O} 2$ & $1.98 \pm 0.14$ & $1.83 \pm 0.11$ & $1.81 \pm 0.10$ & $1.81 \pm 0.10$ & $1.99 \pm 0.14$ & $1.80 \pm 0.12$ & $1.82 \pm 0.10$ & $1.79 \pm 0.11$ & $2.39 \pm 0.21$ \\
\hline $\mathrm{H}^{(\mathrm{O}-\mathrm{Thr} 40)}-\mathrm{O} 2$ & $1.98 \pm 0.14$ & $1.94 \pm 0.13$ & $1.90 \pm 0.14$ & $1.95 \pm 0.13$ & $1.97 \pm 0.14$ & $1.91 \pm 0.12$ & $1.86 \pm 0.11$ & $1.92 \pm 0.13$ & $1.92 \pm 0.11$ \\
\hline
\end{tabular}
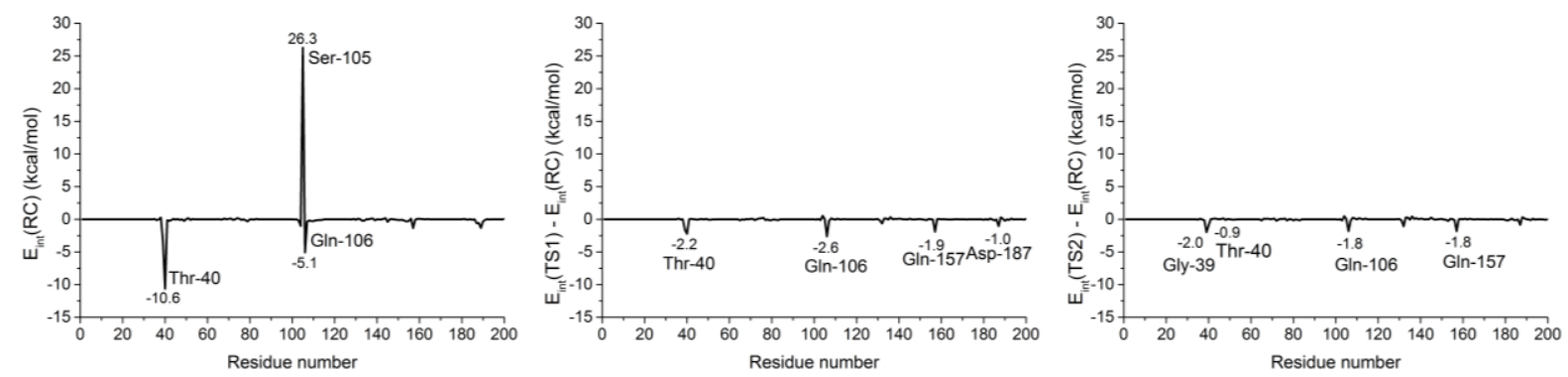

Figure 3. Averaged contributions of individual amino acids to substrate-protein interaction energies (in kcal mol-1), computed for RC, left panel, and difference between TS1 and RC (center panel) and TS2 and RC (right panel).

Distances between the two heavy atoms, OD-ND, is larger in reactants state, intermediate 2 and products state (structures where His224 presents a neutral electronic state) than in the other minimum energy structures. A significantly shorter distance is obtained when His 224 has to reach its protonated state (intermediate 1 and 3), which favors stabilizing the positive charge developed in this residue, and in particular in TS1, where the two residues reach the closest position thus favoring the proton transfer from Ser105.

The other key residues involved in the catalysis are those forming the oxyanion hole; Gln106 and Thr40. As previously proposed in the literature, our calculations show how interactions between the carbonyl oxygen of the substrate and these two residues favor the reaction by polarizing the carbonyl bond. A deeper analysis can be carried out by computing the contributions of individual amino acids to substrate-protein interaction energies in RC, TS1 and TS2 (see Figure 3). This figure, where negative values represent stabilizing effects, confirms the relevance of the residues forming the oxyanion hole in slightly stabilizing the substrate and, in particular, the negative charge developed in $\mathrm{O} 2$ in some of the steps of the full process. Hult and Berglund suggested that the oxyanion hole of PALB form three hydrogen bonds to the oxygen of the substrate in the transition state, whereas, in ground state, only two would be developed. ${ }^{8}$ According to our results, one of the hydrogen bond interactions established with Thr40 is conserved along the reaction path while the other one disappears when arriving at products complex. This could help the substrate to release in the last step. The third hydrogen bond interaction of the oxyanion hole, the one established with Gln106 appears to be much weaker. Interestingly, and in agreement with the proposal of Hult and Berglund, the largest value of the distance describing this interaction (HGln106-O2) is observed in reactants, while in the TS1 and during the intermediate steps this distance is noticeably shortened (from 3.70 to $2.99 \AA$ ). Moreover, and similarly to the behavior of the HGln106-O2 hydrogen bond, once arriving to products, the distance is enlarged again. These distances present their minimum values in intermediate 1 and 3 , where the double bond is transformed into a single bond and the maximum separation of charges is reached.

This behavior is confirmed by inspection of not only averaged distances listed in Table 1 but the atomic charges reported in Table 2. Charge on carbonyl oxygen atom of the substrate (O2) arrives to 1.04 and -1.09 a.u. in I1 and I3, where the maximum positive values on $\mathrm{C} 1$ are also reached; 0.98 and 1.09 a.u., respectively.

From the energetics point of view, the reaction paths traced on the M06/MM and AM1/MM free energy surfaces (Fig. 1 and S2, respectively), have been projected into a two-dimensional free energy profile that is depicted in Figure 4. First conclusion that can be derived is that a dramatic reduction of the free energy barriers of all steps of the process is obtained when improving the QM description of the system from a semiempirical to a density functional theory (DFT) Hamiltonian. In fact, it is noticeable how M06-2X/MM calculations predict a rather short half-life time for intermediate 1 and 3. On the other hand, Intermediate 2 appears to be intensely stabilized by the oxyanion hole and the covalent bond the substrate establishes with Ser105. This result is obtained at both levels of theory, AM1/MM and M06-2X/MM. Consequently; we predict this species could be isolated experimentally. According to the M06-2X/MM results, the highest energy TSs correspond to the first step describing the nucleophilic attack of the serine oxygen to the carbonyl carbon of the ester and the second step where the ester bond of the substrate is broken, with participation of His224. The M06-2X/MM energy of TS1 and TS2 relative to 
Table 2 Averaged atomic charges (in a.u.) obtained in the states located along the reaction path of the hydrolysis of methyl acrylate catalyzed by PALB.

\begin{tabular}{|c|c|c|c|c|c|c|c|c|c|}
\hline & RC & TS1 & I1 & TS2 & 12 & TS3 & I3 & TS4 & PC \\
\hline $\mathrm{C} 1$ & 0.920 & 0.914 & 0.984 & 0.837 & $\begin{array}{l}0.798 \\
\end{array}$ & $\begin{array}{l}1.038 \\
\end{array}$ & 1.091 & 1.010 & 0.892 \\
\hline O1 & -0.358 & -0.420 & -0.605 & -0.386 & -0.455 & -0.493 & -0.533 & -0.468 & -0.609 \\
\hline $\mathrm{O} 2$ & -0.777 & -1.000 & -1.041 & -0.982 & -0.753 & -1.056 & -1.095 & -1.027 & -0.778 \\
\hline Asp187-COO- & -0.842 & -0.839 & -0.841 & -0.849 & -0.846 & -0.848 & -0.847 & -0.840 & -0.836 \\
\hline HD & 0.344 & 0.320 & 0.305 & 0.302 & 0.302 & 0.335 & 0.341 & 0.328 & 0.326 \\
\hline ND & -0.302 & -0.180 & -0.081 & -0.094 & -0.172 & -0.261 & -0.230 & -0.237 & -0.288 \\
\hline OG & -0.352 & -0.272 & -0.512 & -0.448 & -0.420 & -0.594 & -0.663 & -0.604 & -0.608 \\
\hline
\end{tabular}

Table 3. Averaged KIEs computed at AM1/MM level at 300K for the hydrolysis of methyl acrylate in the active site of PALB.

\begin{tabular}{ccccc}
\hline & TS1 & TS2 & TS3 & TS4 \\
\hline${ }^{13} \mathbf{C 1}$ & $1.0081 \pm 0.0019$ & $1.0080 \pm 0.0027$ & $1.0010 \pm 0.0040$ & $1.0071 \pm 0.0037$ \\
${ }^{18} \mathbf{O 1}$ & $0.9853 \pm 0.0042$ & $1.0056 \pm 0.0064$ & - & - \\
${ }^{18} \mathbf{O 2}$ & $0.9911 \pm 0.0066$ & $0.9805 \pm 0.0075$ & $0.9795 \pm 0.0094$ & $0.9815 \pm 0.0091$ \\
\hline
\end{tabular}

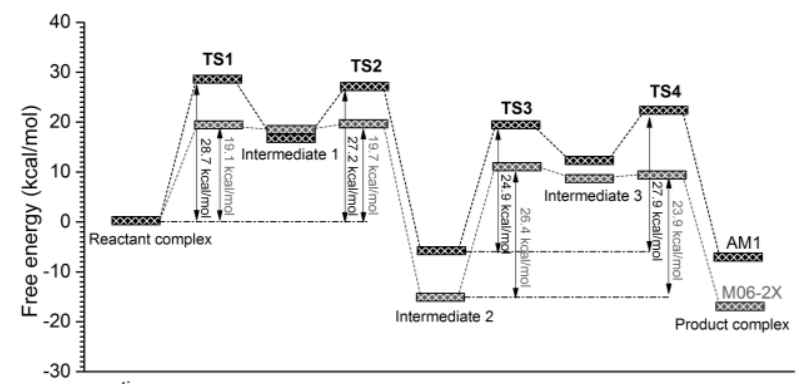

Figure 4. Free energy profile for the multi-step reaction mechanism of hydrolysis of methyl acrylate catalyzed by PALB obtained at M06-2X/OPLS/TIP3P (in grey) and AM1/OPLS/TIP3P (in black).

reactants is very close, 19.1 and $19.7 \mathrm{kcal} \cdot \mathrm{mol}^{-1}$, respectively. This value is in very good agreement with the $20.3 \mathrm{kcal} \cdot \mathrm{mol}^{-1}$, which is the free energy barrier that can be deduced from the experimental catalytic events measure by Berglund and co-workers. ${ }^{15}$ TS3, associated to the nucleophilic attack of the water molecule to the carbonyl carbon of the substrate, presents the highest energy barrier at this level of calculation. Nevertheless, it is important to note that energy of TS1 and TS2 are clearly higher than TS3. The higher barrier of third step is due to the strong stabilization of Intermediate 2 and also to a possible overestimation due to the procedure followed to perform the energy corrections at M06-2X level. In fact, as previously shown in our study of the Michael-type addition performed in gas phase by means of the AM1 and by DFT calculations within the B3LYP and M06-2X hybrid functionals, no displacement was observed of the proton between the dyad formed by His 224 and Asp187 at AM1 level when a positive charge is developed in the His $224 .{ }^{16}$ This is in agreement with the present QM/MM results, as deduced from Table 1. On the contrary, a spontaneous transfer of the proton to the aspartate can take place when using DFT methods, thus stabilizing the system. Since the corrected high level 2D PMFs surfaces have been obtained from the structures generated from the AM1/MM calculations, no possible exploration of a lower energy TS can be performed at M06-2X/MM.

In order to support the proposed mechanism as well as to confirm which is the rate-limiting step, kinetic isotope effects (KIE) could be measured. Thus, 10 different structures of TSs and reactants have been selected from the constrained MD simulations performed on these states in order to compute KIEs for substitution on key atoms $\mathrm{C} 1, \mathrm{O} 1$ and $\mathrm{O} 2$. Analysis of results listed in Table 3 shows that isotopic substitution of $\mathrm{C} 1$ and $\mathrm{O} 2$ does not render differences between TS1 and TS3. Obviously, the change in orbital hybridization of these two atoms in step 1 and 3 is equivalent; $\mathrm{C} 1$ changes from $\mathrm{sp} 2$ to $\mathrm{sp} 3$, and $\mathrm{O} 1$ changes from double bond to single bond. Mass substitution on $\mathrm{O} 1$ could shed some light into the question. According to the four-steps mechanism, and since KIEs are computed (and measured) as a relative effect between each of the TS and reactants (Michaelis Complex), very slightly inverse effect should be expected if TS1 were the rate limiting step ( 0.9853 \pm 0.0042 ). Nevertheless, a measurable normal effect would be in case of TS2, when $\mathrm{O} 1-\mathrm{C} 1$ bond is broken and a proton is transfer to O1 from His224 $(1.0056 \pm 0.0064)$. Since O1 belongs to the methanol molecule that is released in step 2, no calculations have been performed for steps 3 and 4 .

Finally, an alternative reaction mechanism has been explored, where the reaction can proceed in two steps through an intermediate equivalent to the intermediate 2 located in the previously discussed mechanism. A schematic representation of the stationary point structures is presented in Figure 5 while the free energy profile, computed at AM1/MM and M06-2X/MM level, is shown in Figure 6. As observed, the attack of Ser105 and hydrolysis of substrate takes place in a single concerted step. The second step corresponds to the nucleophilic attack of the water molecule and the scission of the bond established between the substrate and Ser105 to release products and regenerate the protein. The results suggest that the four-steps mechanism is kinetically more favorable than this twosteps mechanism, considering that significantly lower energy barriers are obtained for the former.

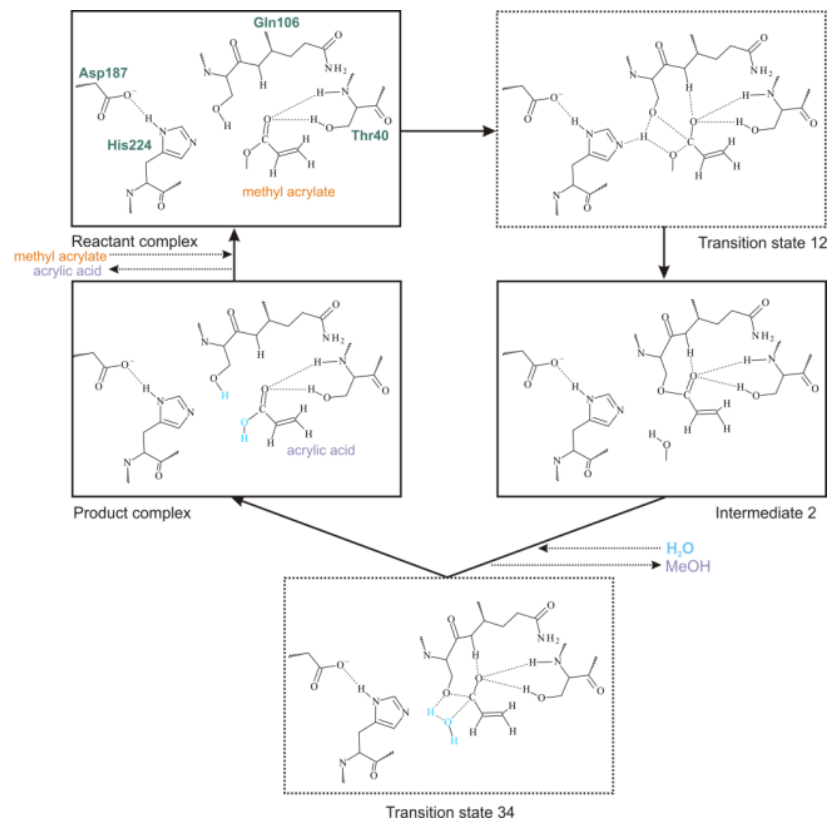

Figure 5. Two-step proposed reaction mechanism for hydrolysis of methyl acrylate catalyzed by PALB. 


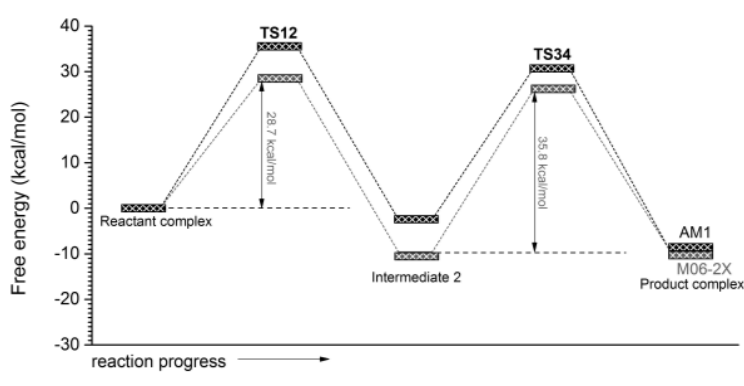

Figure 6. Free energy reaction pathway of the two steps mechanisms of hydrolysis of methyl acrylate catalyzed by PALB computed at AM1/MM (black line) and M062X/MM (grey line) level.

\section{Conclusion}

The study of the primary reaction of promiscuous Pseudozyma (formerly Candida) antarctica lipase B (PALB), its native hydrolytic activity on methyl acrylate molecule, has been studied using hybrid $\mathrm{QM} / \mathrm{MM}$ MD simulations describing the QM region at two levels of theory, AM1 and M06-2X. PESs and free energy surfaces have been explored and the results suggest that the catalytic reaction can take place by means of a four-step mechanism equivalent to the pingpong bi-bi reaction mechanism initially proposed by Hult and Berglund. ${ }^{12,14} \mathrm{~A}$ possible two-steps mechanism has been also explored but the resulting free energy barriers are much higher. According to our results the first step, the activation of the carbonyl group of the substrate and the nucleophilic attack of Ser105 to the carbonyl carbon atom, presents the highest energy transition state, while the dramatic stabilization of intermediate 2 , which correspond to the hydrolyzed acrylate molecule covalently bonded to the protein backbone, provokes that the third step, the nucleophilic attack of a conserved water molecule located in the active site, presents the highest energy barrier. It is important to point out the significant effect on the energetics of the reaction when improving the QM description of the system from semiempirical to DFT. Thus, a dramatic reduction of the free energy barriers of all steps of the process is observed at M06-2X/MM level by comparison with the AM1/MM results. Also, it is noticeable how M06-2X/MM calculations predict a rather short half-life time for intermediate 1 and 3. On the other hand, Intermediate 2 appears to be a very stable intermediate stabilized by the oxyanion hole and the covalent bond the substrate establishes with Ser105. Consequently; we predict this species could be isolated experimentally. According to the M062X/MM results, the highest energy TSs correspond to the first step describing the nucleophilic attack of the serine oxygen to the carbonyl carbon of the ester and the second step where the ester bond of the substrate is broken, with participation of His224. The M06-2X/MM energy of TS1 and TS2 relative (19.1 and 19.7 $\mathrm{kcal} \cdot \mathrm{mol}^{-1}$, respectively) is very close to the free energy barrier that can be deduced from the experimental catalytic events measure by Berglund and co-workers $\left(20.3 \mathrm{kcal} \cdot \mathrm{mol}^{-1}\right) .{ }^{15}$

Our results suggest that the origin of the catalytic activity of the enzyme is due to favorable interactions established between the residues of the active site that create the oxyanion hole, Gln106 and Thr40, and the carbonyl oxygen atom of the substrate that develops a negative charge during the reaction and, in particular in the rate limiting TS. Nevertheless, there are other key residues for catalysis such as Ser105 that forms a covalent bond with the substrate stabilizing the intermediate states, or the Asp187 that is capable of modulating the pKa of His224, favoring its role as base or acid depending on the step of the process. KIEs have been computed for substitutions of some key atoms, revealing the possibility of using this tool to support the proposed mechanism and to distinguish the rate-limiting step.

A detailed knowledge of the molecular mechanism of the primary reaction of a promiscuous enzyme can be used in rational designs of new catalysts for organic synthesis based on its protein scaffold. In particular, some of the features of PALB such as the oxyanion hole of the active site, capable of stabilizing a negatively charge that can be develop during the reaction, or the existence of a Asp-His dyad with a modulated pKa can be used to re-design PALB into a catalysts for a different reaction.

\section{Experimental Section}

The System. The starting geometry was obtained from the Candida Antarctica lipase B (PALB) PDB entry 1TCA, ${ }^{17}$ which consists of a monomer. PROPKA3 semiempirical program $^{18}$ was used to recalculate the $\mathrm{pKa}$ values of the titratable protein residues to verify their protonation states at a $\mathrm{pH}$ of 7 . According to the results, most residues were found at their standard protonation state, except for the Asp134 residue that should be protonated and His 224 which was protonated in $\delta$-position. Additionally, the disulphate bridges between Cys22 and Cys64, Cys216 and Cys258, and Cys293 and Cys 311 were defined. Since the total charge of the system was neutral, no counterions were required. After adding the hydrogen atoms to the structure, series of optimization algorithms (steepest descent conjugated gradient and L-BFGS-B) were applied. To avoid a denaturation of the protein structure, all the heavy atoms of the protein and the inhibitor were restrained by means of a Cartesian harmonic umbrella with a force constant of $1000 \mathrm{~kJ} \cdot \mathrm{mol}^{-1} \cdot \AA^{-2}$. Afterward, the system was fully relaxed with the peptidic backbone was restrained with a lower constant of $100 \mathrm{~kJ} \cdot \mathrm{mol}^{-1} \cdot \AA^{-2}$. Finally, the optimized protein was solvated using a orthorhombic box of water molecules of $100 \mathrm{x}$ 80 x $80 \AA$ of side. Water molecules with an oxygen atom lying within $2.8 \AA$ of any heavy atom were removed. The remaining water molecules were then relaxed using optimization algorithms. The full system was formed by the 64652 atoms, containing the protein (4625 atoms), the methyl acrylate substrate (12 atoms), 1 and 20005 solvation water molecules (60015 atoms).

During the simulations, the atoms of the substrate, together with the side chains of His 224 , Asp187 and residue Ser105 were selected to be treated by QM, using the semiempirial AM1 hamiltonian. The rest of the system (protein plus water molecules) were described using the OPLS-AA and TIP3P force fields, respectively, as implemented in the fDYNAMO library. ${ }^{19}$ To saturate the valence of the QM/MM frontier atoms, link atoms between the $\mathrm{C} \alpha$ and $\mathrm{C} \beta$ atoms of the three key residues were used. (see Scheme 1).

Due to the large amount of degrees of freedom, any residue $20 \AA$ apart from any of the atoms of the substrate was kept frozen in the remaining calculations. Cut-offs for the non-bonding interactions were applied using a switching scheme, within a range radius from 14.5 to $16 \AA$. The system was equilibrated by means of $500 \mathrm{ps}$ of QM/MM MD at temperature of $300 \mathrm{~K}$.

The Potential of Mean Force (PMF). In order to obtain the reaction energy profile in terms of free energies, we have traced several PMFs. All PMFs have been calculated using the weighted histogram analysis method (WHAM) ${ }^{20}$ combined with the umbrella sampling approach ${ }^{21}$ as implemented in fDYNAMO. The procedure for the PMF calculation is straightforward and requires series of molecular dynamics simulations in which the distinguished reaction coordinate variable, $\xi$, is constrained around particular values. The values of the variables sampled during the simulations are then pieced together to construct a distribution function from which the PMF is obtained as a function of the distinguished reaction coordinate $(\mathrm{W}(\xi))$. The PMF is related to the normalized probability of finding the system at a particular value of the chosen coordinate by equation (6):

$W(\xi)=C-k T \ln \int \rho\left(r^{N}\right) \delta\left(\xi\left(r^{N}\right)-\xi\right) d r^{N-1}$

The activation free energy can be then expressed as:

$\Delta G^{\ddagger}(\xi)=W\left(\xi^{\ddagger}\right)-\left[W\left(\xi^{R}\right)+G_{\xi}\left(\xi^{R}\right)\right]$

where the superscripts indicate the value of the reaction coordinate at the reactants $(\mathrm{R})$ and TS and $G_{\xi}\left(\xi^{n}\right)$ is the free energy associated with setting the reaction coordinate to a specific value at the reactant state. Normally this last term makes a small contribution and the activation free energy is directly estimated from the PMF change between the maximum of the profile and the reactant's minimum:

$\Delta G^{\ddagger}(\xi) \approx W\left(\xi^{\ddagger}\right)-W\left(\xi^{R}\right)=\Delta W^{\ddagger}(\xi)$

The selection of the reaction coordinate is usually trivial when the mechanism can be driven by a single internal coordinate or a simple combination (as the anti-symmetric combination of two interatomic distances). However this is not the case for all possible steps of the reaction subject of study in this paper where many coordinates are participating. Instead we were compelled to obtain a much more computationally demanding 2D-PMF using two coordinates: $\zeta_{1}$ and $\zeta_{2}$. The 2D-PMF is related to the probability of finding the system at particular values of these two coordinates: 
$W(\xi)=C^{\prime}-k T \ln \int \rho\left(r^{N}\right) \delta\left(\xi_{1}\left(r^{N}\right)-\xi_{1}\right) \delta\left(\xi_{2}\left(r^{N}\right)-\xi_{2}\right) d r^{N-2}$

To estimate the activation free energy from this quantity we recovered one dimensional PMF changes tracing a maximum probability reaction path on the 2D-PMF surface and integrating over the perpendicular coordinate. Computational details of the procedure followed to get the 2D PMF required to explore every single step of the proposed mechanisms are reported in the Supporting Information.

Because of the large number of structures that must be evaluated during free energy calculations, $\mathrm{QM} / \mathrm{MM}$ calculations are usually restricted to the use of semiempirical Hamiltonians. In order to reduce the errors associated to the quantum level of theory employed in our simulations, a new energy function defined in terms of interpolated corrections was used. ${ }^{22}$

$E=E_{A M 1 / M M}+S\left\lfloor\Delta E_{L L}^{H L}\left(\xi_{1}, \xi_{2}\right\rfloor\right.$

where $S$ denotes a two-dimensional spline function, and its argument $\left[\Delta E_{u}^{u}(\xi, \xi]\right.$ is a correction term evaluated from the single-point energy difference between a high-level (HL) and a low-level (LL) calculation of the QM subsystem. AM1 semiempirical Hamiltonian was used as LL method while a density functional theory (DFT) based method was selected for the HL energy calculation. In particular, HL energy calculations were performed by means of the hybrid M06- $2 \mathrm{X}^{23}$ functional using the standard $6-31+\mathrm{G}^{* *}$ basis set. These calculations were carried out using the Gaussian09 program. $^{24}$

Kinetic isotope effects. Finally, average kinetic isotope effects (KIE) have been computed for isotopic substitutions of key atoms, from the transition states and the reactant complex localized at AM1/MM level of theory.

$K I E=\frac{\left(\frac{Q_{L}}{Q_{H}}\right)_{a}}{\left(\frac{Q_{L}}{Q_{H}}\right)_{b}}=\frac{\left(\frac{A_{L} B_{L} C_{L}}{A_{H} B_{H} C_{H}}\right)_{a}^{1 / 2}}{\left(\frac{A_{L} B_{L} C_{L}}{A_{H} B_{H} C_{H}}\right)_{b}^{1 / 2}} \cdot \frac{\left(\frac{M_{L}}{M_{H}}\right)_{a}^{3 / 2}}{\left(\frac{M_{L}}{M_{H}}\right)_{a b}^{3 / 2}} \cdot \frac{Z P E_{a}}{Z P E_{b}} \cdot \frac{\operatorname{Exc}(a)}{\operatorname{Exc}(b)}$

where, $H$ - heavy isotope, $L$ - light isotope, $M$ - mass of isotope, $Q$ - partition function, $A, B, C$ - the principal moments of inertia, $a$ - transition state structure coordinates, $b$ reactant complex structure coordinates, $Z P E$ - the zero point energy factor and Exc Boltzman excitation factors, respectively, using the rigid-rotor/harmonic-oscillator approximation, without the scaling of vibrational frequencies, as explained and applied in previous papers ${ }^{25,26}$ The Hessian was computed for a subset of atoms of the full system, those of the QM region, consistent with the "cut-off rule" and the local nature of isotope effects. ${ }^{27}$

\section{Acknowledgements}

We thank the Spanish Ministerio de Ciencia e Inovación for project CTQ2009-14541 C2, Universitat Jaume I - BANCAIXA Foundation for project P1・1B2011-23, Generalitat Valenciana for Prometeo/2009/053 project and the Polish National Center for Science (NCN), grant 2011/02/A/ST4/00246 (2012-2017). The authors also acknowledge the Servei d'Informatica, Universitat Jaume I, for generous allotment of computer time.

[1] F. H. Arnold, Nature 2001, 409, 253-257.

[2] A. S. Bommarius, J. K. Blum, M. J. Abrahamson, Curr. Opin. Chem. Biol. 2011, $15,194-200$

[3] D. Röthlisberger, O. Khersonsky, A. M. Wollacott, L. Jiang, J. DeChancie, J. Betker, J. L. Gallaher, E. A. Althoff, A. Zanghellini, O. Dym, S. Albeck, K. N. Houk, D. S. Tawfik, D. Baker Nature 2008, 453, 190-195.

[4] J. B. Siegel, A. Zanghellini, H. M. Lovick, G. Kiss, A. R. Lambert, J. L. St.Clair, J. L. Gallaher, D. Hilvert, M. H. Gelb, B. L. Stoddard, K. N. Houk, F E. Michael, D. Baker, Science 2010, 329, 309-313.

[5] K. Świderek, I. Tuñon, V. Moliner, WIREs Comput. Mol. Sci. 2013, doi 10.1002/wcms. 1173

[6] P. J. O'Brien, D. Herschlag, Chem. Biol. 1999, 6, R91-R105.

[7] A. Aharoni, L. Gaidukov, O. Khersonsky, S. McQ Gould, C. Roodveldt, D.S Tawfik, Nat. Genet. 2005, 37, 73-76.

[8] K. Hult, P. Berglund, Trends Biotech. 2007, 25, 231-238

[9] D. L. Ollis, E. Cheah, M. Cygler, B. Dijkstra, F. Frolow, S. M. Franken, M Harel, S. J. Remington, I. Silman, J. Schrag, J. L. Sussman, K. H. G. Verschueren, A. Goldman, Protein Eng. 1992, 5, 197-211.

[10] M. Holmquist, Curr. Protein Pept. Sci. 2000, 1, 209-235.

[11] J. Uppenberg, N. Öhrner, M. Norin, K. Hult, G.J. Kleywegt, S. Patkar, V. Waagem, T. Anthonsen, T.A. Jones, Biochemistry 1995, 34, 16838-16851.

[12] K. Hult, P. Berglund, Trends Biotech. 2007, 25, 231-238.

[13] A. Warshel M. J. Levitt, Mol. Biol. 1976, 103, 227-249.

[14] K. Hult, in Microbial reagents in organic synthesis. (Eds: S. Servi) Kluwer academic publishers, The Netherlands, 1992, pp. 289-298.

[15] M. Svedendahl, B. Jovanovic, L Fransson, P. Berglund, ChemCatChem 2009, 1, $252-258$.

[16] K. Świderek, A. Pabis, V. Moliner, Org. Biomol. Chem. 2012, 10, 5598-5605.

[17] J. Uppenberg, M. T. Hansen, S. Patkar, T. A. Jones, Structure, 1994, 2, 293-308.
[18] a) H. Li, A. D. Robertson, J. H. Jensen, Proteins 2005, 61, 704-721. b) D. C. Bas, D. M. Rogers, J. H. Jensen, Proteins 2008, 73, 765-783. c) M. H. M. Olsson, C. R. Søndergard, M. Rostkowski, J. H. Jensen, J. Chem. Theor Comput. 2011, 7, 525-537. d) C. R. Søndergaard, M. H. M. Olsson, M Rostkowski, J. H. Jensen, J. Chem. Theor. Comput. 2011, 7, 2284-2295.

[19] M. J. Field, M. Albe, C. Bret, F. Proust-De Martin, A. Thomas, J. Comp. Chem. 2000, 21, 1088-1100.

[20] S. Kumar, D. Bouzida, R. H. Swendsen, P. A. Kollman, J. M. Rosenberg, J. Comp. Chem. 1992, 13, 1011-1021.

[21] G. M. Torrie, J. P. Valleau, J. Comp. Phys. 1977, 23, 187-199.

[22] a) J. J. Ruiz-Pernía, E Silla, I. Tuñón, S. Martí, J. Phys. Chem. B 2006, 110 , 17663-17670. b) Y. Y. Chuang, J. C. Corchado, D. G. Truhlar, J. Phys. Chem. A 1999, 103, 1140-1149. c) S. Ferrer, S. Martí, V. Moliner, I. Tuñón, J. Bertran, Phys. Chem. Chem. Phys. 2012, 14, 3482-3489.

[23] a) Y. Zhao, D. G. Truhlar, Theor. Chem. Acc. 2008, 120, 215- 241. (b) Y. Zhao, D. G. Truhlar, Acc. Chem. Res. 2008, 41, 157-167.

[24] M. J. Frisch, G. W. Trucks, H. B. Schlegel, G. E. Scuseria, M. A. Robb, J. R Cheeseman, G. Scalmani, V. Barone, B. Mennucci, G. A. Petersson, H. Nakatsuji, M. Caricato, X. Li, H. P. Hratchian, A. F. Izmaylov, J. Bloino, G. Zheng, J. L. Sonnenberg, M. Hada, M. Ehara, K. Toyota, R. Fukuda, J. Hasegawa, M. Ishida, T. Nakajima, Y. Honda, O. Kitao, H. Nakai, T. Vreven, J. A. Montgomery, Jr., J. E. Peralta, F. Ogliaro, M. Bearpark, J. J. Heyd, E. Brothers, K. N. Kudin, V. N. Staroverov, R. Kobayashi, J. Normand, K Raghavachari, A. Rendell, J. C. Burant, S. S. Iyengar, J. Tomasi, M. Cossi, N. Rega, J. M. Millam, M. Klene, J. E. Knox, J. B. Cross, V. Bakken, C. Adamo, J. Jaramillo, R. Gomperts, R. E. Stratmann, O. Yazyev, A. J. Austin, R. Cammi, C. Pomelli, J. W. Ochterski, R. L. Martin, K. Morokuma, V. G. Zakrzewski, G. A. Voth, P. Salvador, J. J. Dannenberg, S. Dapprich, A. D. Daniels, Ö. Farkas, J. B. Foresman, J. V. Ortiz, J. Cioslowski, and D. J. Fox, Gaussian, Inc., Wallingford CT, 2009.

[25] S. Martí, V. Moliner, I. Tuñón, I. H. Williams, J. Phys. Chem. B 2005, 109, 3707-3710.

[26] S. Martí, V. Moliner, I. Tunón, I. H.Williams, Org. Biomol. Chem. 2003, 1, $483-$ 487.

[27] G. D. Ruggiero, S. J. Guy, S. Martí, V. Moliner, I. H. Williams, J. Phys. Org. Chem. 2004, 17, 592-601.

Received: ((will be filled in by the editorial staff))

Revised: ((will be filled in by the editorial staff)) Published online: ((will be filled in by the editorial staff)) 
Hydrolysis -reaction of promiscuous PALB

Katarzyna Świderek, Sergio Martí, Vicent

Moliner* Page - Page

Theoretical study of primary reaction of Pseudozyma antarctica lipase $B$ as the starting point to understand its promiscuity

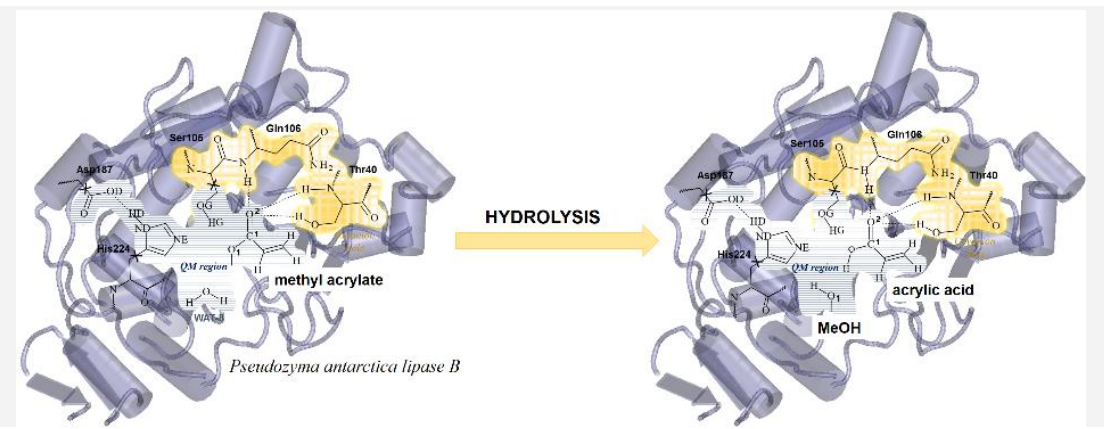

The primary reaction of PALB, its native hydrolytic activity, has been studied using hybrid QM/MM Molecular Dynamics simulations. The free energy surfaces show that the catalytic reaction takes place by means of a multi-step mechanism where the first step, the activation of the carbonyl group of the substrate and the nucleophilic attack of Ser105 to the carbonyl carbon atom, presents the highest energy transition state. The results can be used to rationalize the design of an optimum biocatalyst based on this protein scaffold. 


\section{Supporting Information}

Theoretical study of primary reaction of Pseudozyma antarctica lipase B as the starting point to understand its promiscuity

\section{Katarzyna Świderek ${ }^{1,2}$ Sergio Martí ${ }^{3}$ and Vicent Moliner ${ }^{3, *}$}

\footnotetext{
${ }^{1}$ Departament de Química Física, Universitat de València, 46100 Burjassot, Spain

${ }^{2}$ Institute of Applied Radiation Chemistry, Lodz University of Technology, 90-924 Lodz, Poland.

${ }^{3}$ Departament de Química Física i Analítica, Universitat Jaume I, 12071 Castellón, Spain.
}

Fax: (+34) 964-728066; Tel: (+34) 964-728084; E-mail: moliner@uji.es 


\section{Computational Methods}

The computational details of the procedure followed to get the 2D PMF required to explore every single step of the proposed mechanisms are as follows:

\section{Multi-step mechanism:}

Step 1: 15 simulation windows, in a range of distances from 2.7 to $1.4 \AA$ of the $\mathrm{d}(\mathrm{C} 1-\mathrm{OG})$ coordinate, have been used centered at 61 reference values of the antisymmetric coordinate $\mathrm{d}(\mathrm{OG}-\mathrm{HG})-\mathrm{d}(\mathrm{NE}-\mathrm{HG})$ evenly distributed in a range from -1.5 to $1.5 \AA$.

Step 2: 17 simulation windows, in a range of distances from 1.4 to $3.0 \AA$ of the $\mathrm{d}(\mathrm{C} 1-\mathrm{O} 1)$ coordinate, have been used centered at 41 reference values of the antisymmetric coordinate $\mathrm{d}(\mathrm{NE}-\mathrm{HG})-\mathrm{d}(\mathrm{O} 1-\mathrm{HG})$ evenly distributed in a range from -1.0 to $1.0 \AA$.

Step3: 18 simulation windows, in a range of distances from 3.0 to $1.4 \AA$ of the $\mathrm{d}(\mathrm{C} 1-\mathrm{OW})$ coordinate, have been used centered at 81 reference values of the antisymmetric coordinate $\mathrm{d}(\mathrm{OW}-\mathrm{HW})-\mathrm{d}(\mathrm{NE}-\mathrm{HW})$ evenly distributed in a range from -2.0 to $2.0 \AA$.

Step4: 17 simulation windows, in a range of distances from 1.4 to $2.5 \AA$ of the $\mathrm{d}(\mathrm{C} 1-\mathrm{OG})$ coordinate, have been used centered at 61 reference values of the antisymmetric coordinate d(OG-HW) $-\mathrm{d}(\mathrm{NE}-\mathrm{HW})$ evenly distributed in a range from -1.5 to $1.5 \AA$.

\section{Two-step mechanism:}

Step 1: 27 simulation windows, in a range of distances from -1.3 to $1.3 \AA$ of the antisymmetric d(C1-O1) $-\mathrm{d}(\mathrm{C} 1-\mathrm{OG})$ coordinate, have been used centered at 61 reference values of the antisymmetric coordinate $\mathrm{d}(\mathrm{OG}-\mathrm{HG})-\mathrm{d}(\mathrm{O} 1-\mathrm{HG})$ evenly distributed in a range from -1.5 to $1.5 \AA$.

Step 2: 37 simulation windows, in a range of distances from -1.6 to $1.8 \AA$ of the antisymmetric d(OG-C1) $-\mathrm{d}(\mathrm{C} 1-\mathrm{O} 1)$ coordinate, have been used centered at 101 reference values of the antisymmetric coordinate $\mathrm{d}(\mathrm{OW}-\mathrm{HW})-\mathrm{d}(\mathrm{OG}-\mathrm{HW})$ evenly distributed in a range from -2.5 to $2.5 \AA$.

Umbrella force constant of $5000 \mathrm{kcal} \mathrm{mol}^{-1} \AA^{-2}$, were applied to the distinguished reaction coordinates to allow a perfect overlapping among the windows. The weighted histogram analysis method, combined with the umbrella sampling approach, ${ }^{1,2}$ was used to scan the reaction coordinates. 5 ps of relaxation and $10 \mathrm{ps}$ of production, with a time step of $0.5 \mathrm{fs}$, using the velocity Verlet algorithm ${ }^{3}$ to update the velocities, were run in each window. The PMFs were performed at $300 \mathrm{~K}$, using the NVT ensemble. Structures from the previously obtained QM/MM PESs were used as starting points of each window. A switched cut- off from 14.5 to $16 \AA$ was used for all nonbonded MM interactions.

\section{References}

1. S. Kumar, D. Bouzida, R. H. Swendsen, P. A. Kollman, J. M. Rosenberg, J. Comput. Chem. 1992, $13,1011$.

2. G. M. Torrie, J. P. Valleau, J. Comput. Phys. 1977, 23, 187.

3. L. Verlet, Phys. Rev. 1967, 159, 98. 


\section{Multi-step proposed reaction mechanism}
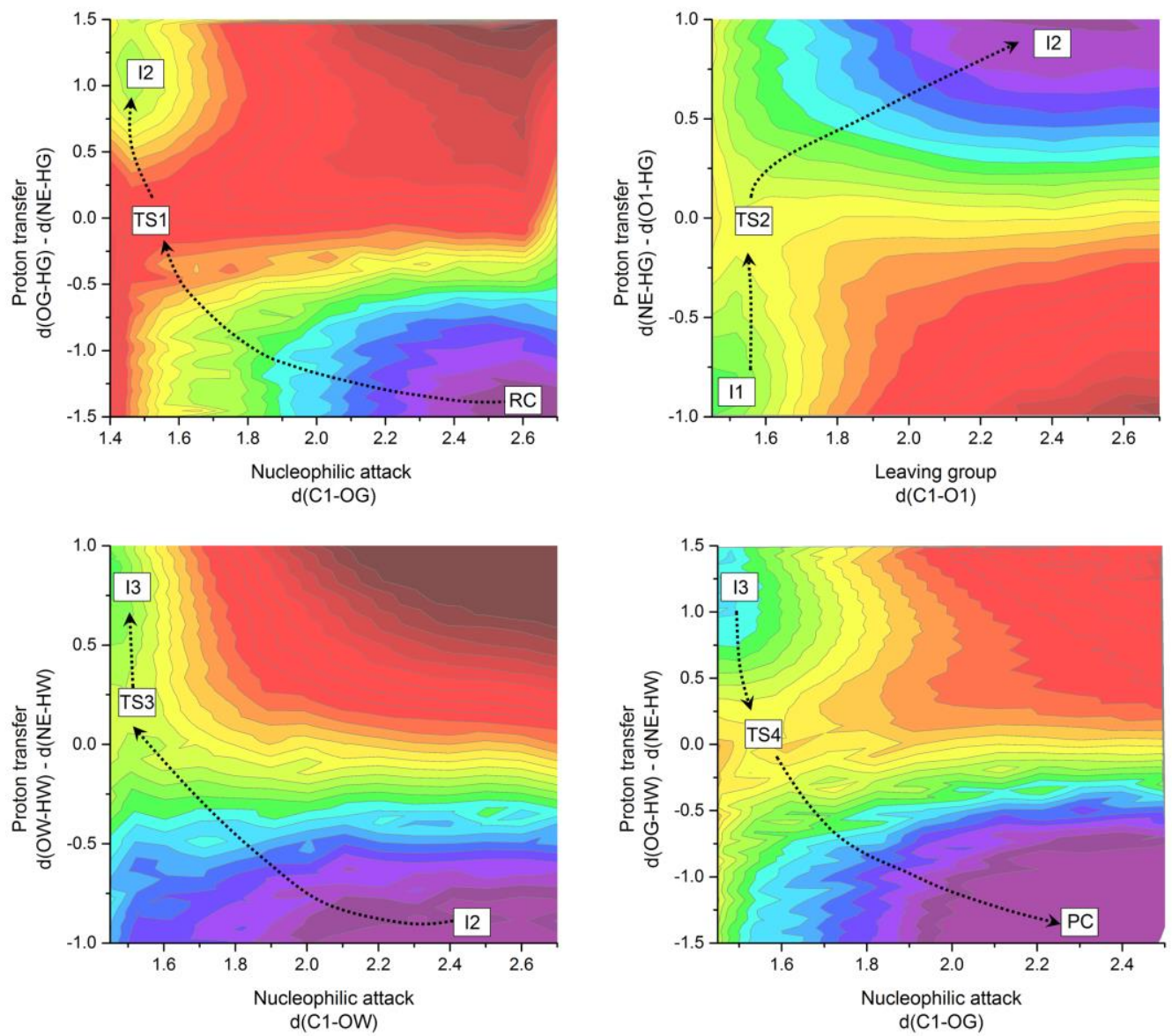

Two-step proposed reaction mechanism
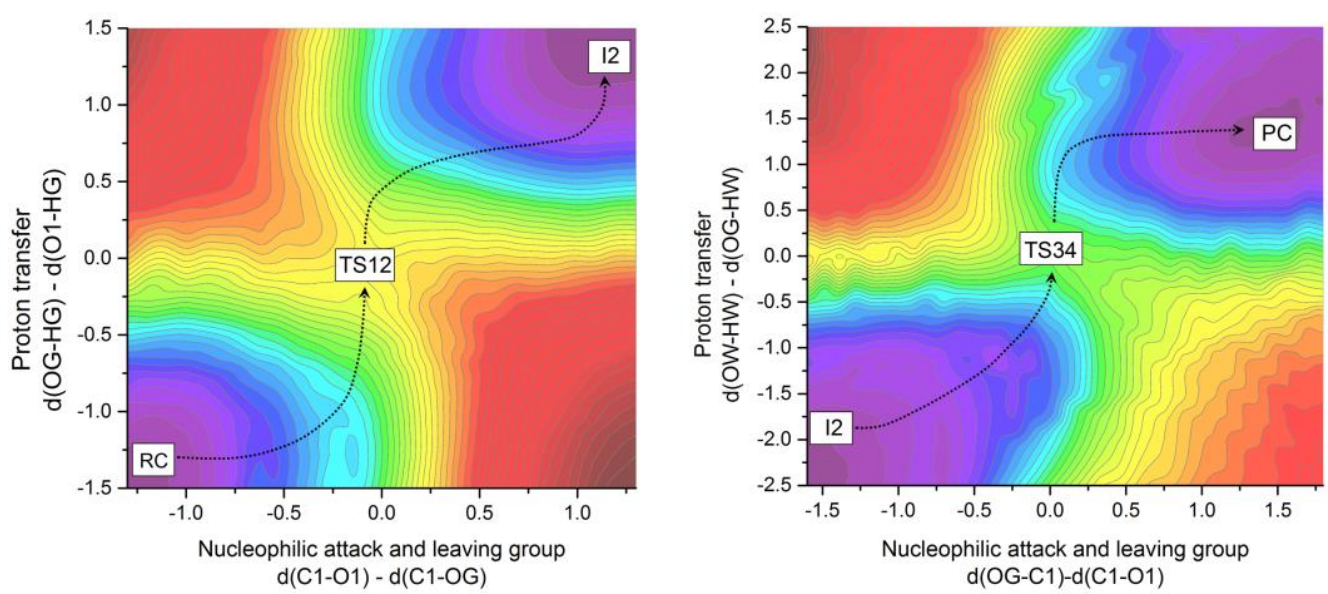

Figure S1. AM1/OPLS/TIP3P PESs for the reaction mechanism of hydrolysis of methyl acrylate catalyzed by PALB. 


\section{Multi-step proposed reaction mechanism}
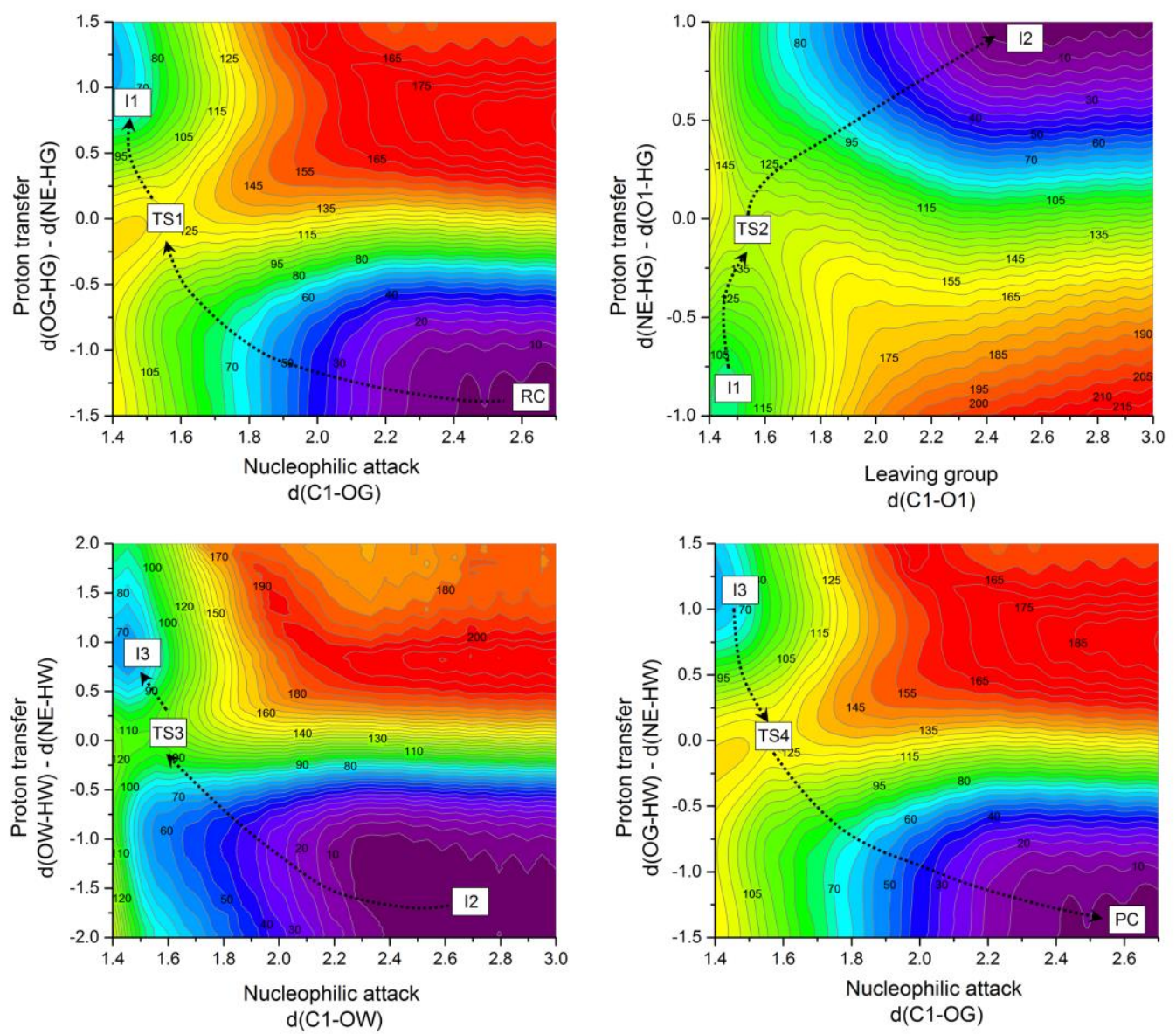

\section{Two-step proposed reaction mechanism}
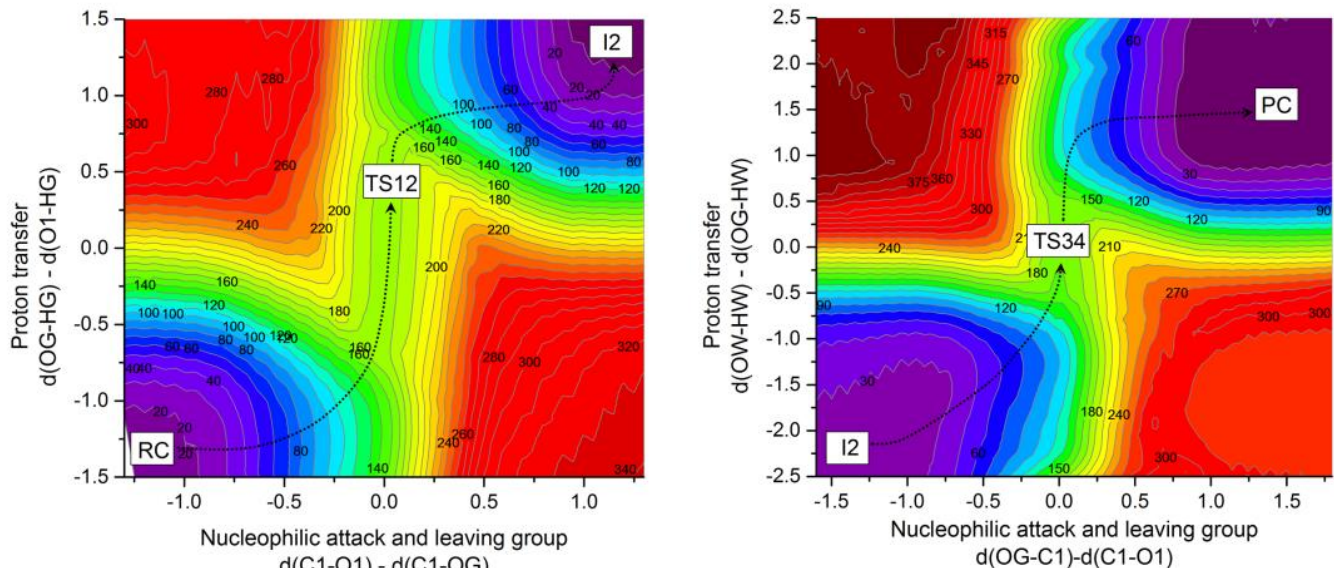

Figure S2. AM1/OPLS/TIP3P 2D-PMF surfaces for the reaction mechanism of hydrolysis of methyl acrylate catalyzed by PALB. 